Monográfico / Monographic

\title{
Género, clase y emancipación: una lectura feminista de Erik Olin Wright
}

\author{
Gender, class and emancipation: A feminist reading of Erik Olin \\ Wright's work
}

\author{
Inés Campillo Poza \\ Universidad Complutense de Madrid, España \\ ines.campillo@ucm.es
}

Recibido / Received: 27/07/2020

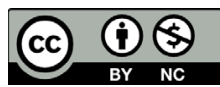

Aceptado / Accepted: 02/12/2020

\section{RESUMEN}

La obra de Erik O. Wright puede entenderse como una renovación del marxismo sociológico, una tradición que ha mantenido un "matrimonio mal avenido" con el feminismo, por usar la conocida expresión de Hartmann. Este artículo se propone examinar el trabajo de Wright desde una perspectiva feminista. En particular, analiza su reflexión teórica en torno a estos dos problemas: por un lado, la intersección de clase y género en el capitalismo contemporáneo y, por el otro, las vías para la emancipación de las mujeres, prestando especial atención a su idea de una sociedad sin género y a los potenciales efectos de "utopías reales" como los permisos parentales iguales e intransferibles. El objetivo del artículo no es sólo revisar la obra de Wright desde un punto de vista feminista, sino explorar el modo en que su contribución entronca con dilemas y debates del feminismo contemporáneo.

Palabras clave: clase, género, interseccionalidad, utopías reales, Erik Wright.

\section{ABSTRACT}

Erik O. Wright's work can be interpreted as an attempt to renovate sociological Marxism, a tradition that has maintained an unhappy marriage' with feminism, to use Hartmann's well-known expression. This paper aims to examine Wright's work from a feminist perspective. In particular, it discusses Wright's theoretical reflection on two problems: on the one hand, the intersection of class and gender in contemporary capitalism; and, on the other, the route to women's emancipation, paying special attention to his idea of a genderlessness society and to the potential effects of 'real utopias' such as equal nontransferrable parental leaves. The aim of the article is not only to review Wright's work from a feminist perspective, but to explore the ways through which his contribution connects with dilemmas and debates of contemporary feminism.

Keywords: class, gender, intersectionality, real utopias, Erik Wright.

Sugerencia de cita / Suggested citation: Campillo, I. (2021). Género, clase y emancipación: una lectura feminista de Erik Olin Wright. Revista Española de Sociología, 30 (2): a41. https://doi.org/10.22325/fes/res.2021.41 


\section{INTRODUCCIÓN}

La obra de Erik Olin Wright es una referencia clave en la sociología y el marxismo contemporáneos. De hecho, sus trabajos sobre análisis de clase y utopías reales pueden verse como un intento de responder a cuestiones centrales de la tradición marxista con ayuda de la investigación sociológica. Como es sabido, sin embargo, el marxismo ha mantenido un "matrimonio mal avenido" (Hartmann, 1979) con el feminismo. La razón principal es que desde el primero se ha tendido, con frecuencia, a subordinar la relevancia de las relaciones de género a los conflictos de clase o el desarrollo del capitalismo, ignorando su autonomía y especificidad. Pero las tensiones de este infeliz matrimonio se han expresado, sobre todo, en el terreno de la práctica, donde esas consideraciones teóricas se han traducido en una supeditación de las reivindicaciones de las mujeres a las necesidades de la lucha de clases.

Wright era bastante consciente de estas circunstancias y del modo en que podian afectar a su propio trabajo. En uno de sus libros reflexiona sobre el sesgo de género del September Group de marxistas analíticos del que formaba parte: "sin duda, la composición de género de la red refleja el papel marginado de las intelectuales en la tradición marxista y contribuye de alguna manera a sostener tal desigualdad de género" (Wright, 1994b, p. 15). Es probable que este estado de cosas guarde relación con el escaso impacto que ha tenido la obra de Wright (y de otros marxistas) en el feminismo contemporáneo.

Sin embargo, la desatención no es recíproca. El feminismo, y especialmente el de corte socialista, sí tuvo una influencia clara en la trayectoria intelectual de Wright, probablemente mayor que la que ejerció sobre el resto de marxistas analíticos. De hecho, el Comparative Class Analysis Project, dirigido por Wright entre los años ochenta y la primera mitad de los noventa, fue no sólo el primer estudio comparativo internacional sobre estructura de clase y conciencia de clase, sino que también fue pionero en el análisis de la interacción entre desigualdades de clase y de género en fenómenos como la brecha de género en las posiciones de autoridad en el trabajo y el techo de cristal (Wright et al., 1995; Wright, 2000; Wright y Baxter, 2000; Baxter y Wright, 2000), el reparto de las tareas domésticas y de cuidados (Wright et al., 1992; Wright, 2000), y la conciencia de clase (Wright, 1989, 2000).

Pero el feminismo también ejerció una influencia menos visible. Como ha subrayado su colega y amigo íntimo Michael Burawoy (2020), la trayectoria intelectual de Wright giró en torno a "dos marxismos": el marxismo "científico" como análisis de clase y el marxismo "crítico" como teoría emancipadora. Wright dedicó el grueso de la primera parte de su trayectoria -desde los años setenta hasta los noventa- a la renovación del análisis de clase para adaptarlo a las sociedades capitalistas avanzadas; y a partir de entonces se dedicó fundamentalmente al problema de las alternativas emancipadoras al capitalismo a través de The Real Utopias Project. En su intervención en uno de los homenajes que se han rendido a Wright ${ }^{1}$, su colega y amiga Gay Seidman (2020, p. 2) recuerda que este viraje "desconcertó" a muchos de sus colegas y amigos. Wright explicaba que el giro hacia la reflexión sobre la utopía estuvo motivado por la caída del muro de Berlín, la desintegración de la Unión Soviética y el triunfo del neoliberalismo (Wright, 2014, p. 8); pero Seidman (2020) subraya que ese cambio también "estuvo moldeado por un profundo compromiso con las preocupaciones feministas" (p. 5).

Tirando de esos hilos, este artículo ofrece una revisión de la obra de Wright desde una perspectiva feminista y se propone explorar los modos en que su contribución conecta con

\footnotetext{
1 En el Congreso Internacional "Transforming Capitalism through Real Utopias: Advancing Erik Olin Wright's Legacy", que se celebró en Coimbra en enero de 2020. En "Celebrating the Life and Work of Erik Olin Wright", su amigo Michael Burawoy está recopilando la información de los distintos homenajes, así como los textos y vídeos de algunas de las intervenciones (ver: https:// thelifeandworkoferikolinwright.wordpress.com/).
} 
algunos debates y reflexiones feministas actuales. Como veremos, su trayectoria intelectual -tanto en el análisis de clase como en las utopías reales- podría leerse en términos de una creciente, aunque irregular, atención al género y a las contribuciones feministas. En buena medida, Wright estaba convencido de que marxismo y feminismo no estaban abocados al divorcio, sino que podían formar un matrimonio más saludable.

El artículo está organizado en dos largos apartados que se corresponden con los programas de investigación mencionados. En el primero, se aborda el lugar que ocupó el género en la renovación del análisis de clase de Wright y su aportación al estudio de las interacciones entre clase y género. En el segundo, se examina la influencia del feminismo en su reformulación de una alternativa emancipadora y sus intervenciones en los debates sobre la igualdad.

\section{ANÁLISIS DE CLASE Y RELACIONES DE GÉNERO}

El programa de investigación en torno al análisis de clase de Wright surge de la necesidad de adaptar el mapa abstracto y polarizado de clases de Marx a las sociedades capitalistas avanzadas, en las que se había producido una expansión tanto de las ocupaciones profesionales y técnicas como de los puestos de supervisión y dirección. Se trataba, según sus propias palabras, de dar respuesta al "engorro de las clases medias" (Wright, 1994a, p. 10). A su juicio, la adaptación del mapa de clases marxiano era una condición necesaria para el estudio de los procesos contemporáneos de formación y conflicto de clase: sin comprender correctamente la estructura de clase no se podía entender ni anticipar la formación de actores políticos de clase, así como tampoco las posibles alianzas o conflictos que podían acercarnos al camino hacia el socialismo. Por esa razón, el trabajo de Wright se centró fundamentalmente en esa primera tarea.

Wright define la estructura de clases como "la estructura de relaciones sociales en la que están inmersos los individuos (o, en algunos casos, las familias), y que determinan sus intereses de clase" (Wright, 1994a, p. 5). La idea clave es que "lo que tienes determina lo que obtienes y lo que tienes que hacer para obtenerlo" (Wright, 2005, p. 22). Esa estructura "define un conjunto de huecos o posiciones que son ocupadas por los individuos o las familias" (Wright, 1994a, p. 5). Esas posiciones no tienen por qué ser univocas, especialmente en las sociedades capitalistas avanzadas en las que no sólo no han desaparecido las "viejas" clases medias (la pequeña burguesía), sino que ha crecido una amplia "nueva" clase media asalariada.

La principal contribución de Wright al análisis de clase es su teorización de ese rompecabezas por medio de la idea de "posiciones de clase contradictorias": posiciones con "un carácter múltiple" y que "son contradictorias precisamente en el sentido de que participan en los dos bandos [explotadores y explotados] en este conflicto de intereses inherentemente contradictorios" (Wright, 1994a, pp. 49-50). Aunque esta idea conoció diferentes formulaciones (Wright, 1983, 1994a), la última de ellas se fundamentaba en la conceptualización roemeriana de explotación (Roemer, 1989) y distinguía tres tipos de mecanismos de explotación, según se basaran en la posesión de bienes de producción, bienes de control organizativo o bienes de cualificación. La posición contradictoria de las nuevas clases medias se definía precisamente por su posesión de alguno de estos dos últimos tipos de bienes.

La contribución de Wright al estudio de las interconexiones entre clase y género parece haber pasado más inadvertida y ha generado menos debates. No obstante, como se ha señalado, su renovación del análisis de clase estuvo marcada por una creciente atención a los debates feministas y sus críticas al estudio de la estratificación social desde una 
perspectiva de género. Esa atención se plasmaría, a lo largo de los años, tanto en la reflexión teórica acerca de la interacción entre las relaciones de clase y las de género, como en el estudio empírico de determinados fenómenos (la brecha de género en las posiciones de autoridad en el trabajo, el techo de cristal, el reparto de las tareas domésticas y de cuidados en los hogares o la conciencia de clase) que representaban un ejemplo de tales interconexiones.

Las primeras ideas de esa atención teórica al género pueden rastrearse en sus primeras obras, en las que aparece un cuestionamiento, todavía poco sistemático, del análisis de clase marxista tradicional desde una perspectiva de género y un cierto interés por los debates del feminismo socialista. Si a finales de los setenta la mayor parte de los marxistas (y de los sociólogos dedicados a la estratificación social) argumentaban que la familia era el elemento constitutivo apropiado de la clase, en Class Structure and Income Determination Wright (1979) defendía que "si son los individuos o las familias los elementos apropiados de la clase es en parte una cuestión histórica, no simplemente una cuestión teórica a priori. [...] Pero, en general, especialmente en el capitalismo avanzado, es más apropiado entender que son los individuos los que ocupan posiciones de clase" (p. 210). De hecho, en esta obra estudia a las mujeres trabajadoras "como individuos insertos en los espacios vacíos de las relaciones sociales de producción más que como miembros de unidades familiares dentro de las relaciones sociales de reproducción" (Wright, 1979, p. 211). No obstante, esta apuesta temprana por un "modelo individualista" del análisis de clase (Albright, 2008) responde a una conveniencia metodológica más que a una postura teórica o un alineamiento claro con la crítica que habían empezado a hacer algunas sociólogas feministas al análisis de clase tradicional (de hecho, no se cita el trabajo de autoras como Acker, 1973).

Más adelante, en Clases Wright (1994a) abordará, aún de forma poco sistemática, la discusión en torno a la relación entre estructura de clases y relaciones de género, en respuesta a tres cuestiones fundamentales: la adquisición y distribución diferencial de bienes de explotación de hombres y mujeres en la fuerza de trabajo; la posición de clase de las amas de casa; y la posibilidad de que las mujeres constituyan una clase. Con respecto a la primera cuestión, señala que las relaciones de género pueden entenderse como uno de los mecanismos que ayudan a explicar la distribución de los bienes de explotación entre las personas: "En algunas sociedades, a las mujeres se las excluye sistemáticamente de toda posibilidad de poseer los bienes de explotación claves; en otras no se les prohíbe legalmente esa posesión, pero las relaciones de género interponen serios obstáculos mediante sistemas de herencia, procesos para la obtención de credenciales, prácticas de promoción de directivos, etcétera" (Wright, 1994a, p. 145). De hecho, en la parte empírica de esa obra constatará que tanto en Estados Unidos como en Suecia la distribución de clase de hombres y mujeres difiere sustantivamente, constituyendo éstas la mayoría de la clase obrera.

Con respecto al debate marxista sobre la posición de clase de las amas de casa, no sujetas directamente a las relaciones de producción, pero inmersas, como habían subrayado algunas autoras feministas, en relaciones "de clase" en el modo de producción doméstico (Delphy, 1984), Wright (1994a) concluye que "las amas de casa de los obreros están en la clase obrera en su relación con el capital y en una variedad de clases posibles respecto de sus maridos" (p. 148). Esto es, frente a algunas teóricas feministas socialistas, Wright no cree que las mujeres estén necesariamente explotadas por sus maridos en el modo de producción doméstico, sino que "la evaluación de esto último depende de cuáles sean las relaciones reales de control dentro de la familia sobre los bienes, los ingresos y el tiempo de trabajo" (Wright, 1994a, p. 148). Como vemos, en estas dos primeras obras no hay aún un posicionamiento claro por un modelo determinado de incorporación de las mujeres al análisis de clase, sino una estrategia metodológica alterna, y no teorizada sistemáticamente, que opta por tomar como unidad de análisis la ocupación de las 
mujeres, cuando estas forman parte de la fuerza de trabajo, o la familia, cuando estas están excluidas de ella.

Por último, Wright discute la posición de ciertas feministas radicales que defienden que las mujeres constituyen una clase. Aunque entiende que esta apuesta tiene mucho que ver con el tradicional desprecio marxista a otras formas de opresión distinta de la clase, sostiene que sólo sirve para "oscurecer la especificidad de la opresión femenina" y "reducir la coherencia teórica del concepto de clase" (Wright, 1994a, p. 149). Si la clase hace referencia a la posición que se ocupa en las relaciones de producción, las mujeres no pueden constituir una clase. Que haya opresión, argumenta, no implica que las mujeres sean una clase.

A partir de la segunda mitad de los ochenta, la perspectiva de género adquirirá una mayor importancia en su obra: Wright ahondará en una teorización sistemática de las relaciones entre clase y género que someterá a prueba empírica a través de las siguientes oleadas del Comparative Class Analysis Project. Como relata Seidman (2020), es probable que en esa reorientación tuviera mucho que ver su trato con colegas feministas, así como con estudiantes de doctorado que le presionaron para que incluyera a teóricas feministas en sus seminarios sobre teoría sociológica². Pero también estuvo motivada por el llamado "debate Goldthorpe" acerca de la mejor estrategia para incorporar a las mujeres al análisis de clase.

El debate se produjo en respuesta a la defensa que hizo John Goldthorpe de la visión convencional. Desde los años setenta se habian multiplicado las críticas feministas a la investigación sobre estratificación social por el "sexismo intelectual" de sus principales supuestos teóricos (Acker, 1973): que la familia es la unidad en el análisis de clase y que el carácter de clase de esta viene determinado por el trabajo del (varón) cabeza de familia. De este modo, la posición de clase de las mujeres casadas derivaba de la de sus maridos. Según las críticas feministas, estos supuestos oscurecían tanto las relaciones de desigualdad de género (en el acceso a recursos, oportunidades, prestigio, etc.) que se daban en el interior de la familia como el hecho de cada vez más mujeres casadas formaran parte de la fuerza de trabajo y de que, por tanto, hubiera más familias heterogéneas en términos de clase.

Así, frente al enfoque tradicional, en los años ochenta comienzan a desarrollarse enfoques alternativos para el análisis de clase: principalmente, un "modelo individualista", que toma al individuo como unidad de análisis y clasifica a hombres y mujeres atendiendo a su propia ocupación, aunque no estén en la fuerza de trabajo en ese momento (Stanworth, 1984); y un "modelo de clasificación conjunta" (Heath y Britten, 1984), que toma como unidad de análisis la familia, pero deriva su carácter de clase de la combinación de los estatus ocupacionales de ambos miembros de la pareja ${ }^{3}$.

Para Goldthorpe $(1983,1984)$, sin embargo, el enfoque convencional no implicaba la negación o la justificación de la desigualdad de género, sino más bien su constatación: "la familia es la unidad de estratificación ante todo porque sólo algunos miembros de la familia, predominantemente varones, tienen, como resultado de su participación laboral, lo que podría denominarse una posición directamente determinada en la estructura de clase. Otros miembros de la familia, incluyendo las esposas, no suelen tener iguales oportunidades para tal participación, y su posición de clase está, así, indirectamente determinada: es decir, 'se deriva' de la del cabeza de familia" (Goldthorpe, 1983, p. 468).

2 Según cuenta Seidman (2020), Wright reaccionó pidiéndole a las estudiantes que diseñaran una sección completa de su curso y propusieran lecturas, y ésa fue la semilla de un grupo de mujeres que aún se reúne 40 años después. Desde ese momento y durante toda su trayectoria, Wright mantuvo debates continuados con académicas feministas (Nancy Folbre, Juliet Schor, Janet Gordon), algunas de las cuales fueron alumnas suyas de doctorado en la Universidad de Madison-Wisconsin (Julia Adams, Greta Krippner, Elizabeth Wrigley-Fields).

3 Para una revisión de las distintas estrategias y los dilemas sin resolver en esta controversia, ver Albright (2008). 
El reconocimiento de la creciente participación laboral de las mujeres casadas y su menor dependencia económica de sus maridos era compatible, según Goldthorpe, con la constatación de que ese empleo formaba parte habitualmente de una estrategia familiar.

La prueba de ello estaría en la habitual discontinuidad de la participación laboral de aquellas, que entran y salen del mercado de trabajo no sólo por cuestiones de crianza, sino dependiendo de los hitos laborales de sus maridos. Considerar que el carácter de clase de una familia podía modificarse con cada cambio en la situación de empleo de las mujeres casadas implicaba, para Goldthorpe, atacar dos de los principios clave del análisis de clase: que las clases definen "colectividades relativamente estables", esto es, grupos que comparten intereses materiales comunes que no se derivan únicamente de los estatus ocupacionales individuales -como parece dar por hecho el modelo individualista, y en menor medida, el de clasificación conjunta-, sino de la capacidad de consumo y del estilo de vida; y que estas colectividades son "la base del conflicto y la movilización sociopolíticos" (Goldthorpe, 1984, p. 491).

En su intervención en ese debate, Wright (1989) parte del reconocimiento de que las familias heterogéneas en términos de clase son una realidad cada vez más extendida en el capitalismo contemporáneo y que, por tanto, el análisis de clase debe afrontar el problema de cómo clasificarlas. Dicho problema es central porque "plantea cuestiones fundamentales sobre la lógica explicativa subyacente del análisis de clase" (Wright, 1989, p. 37): ¿en virtud de qué mecanismos la posición de clase de una persona explica algo: como resultado de sus experiencias en el trabajo -como se deduce del modelo individualista- o debido a los intereses materiales que se suponen comunes a la familia-como se deriva del enfoque convencional-?

Wright propone una original solución conceptual mixta a este dilema: la posición de clase de las personas depende no sólo de las relaciones directas que tienen con el proceso de producción (la ocupación y la posesión de recursos productivos), sino también de las relaciones indirectas o "mediadas" que mantienen con él (a través de sus vínculos familiares). El peso de cada tipo de relación es variable e incierto a priori: "puede haber variaciones tanto dentro como entre estructuras de clase en la importancia relativa que adquieren los diferentes mecanismos que vinculan a la gente a los recursos productivos" (Wright, 1989, p. 42).

En resumen, frente a la posición extrema de Goldthorpe, que sostiene que la posición mediada de las mujeres casadas prima siempre sobre su posición directa, Wright defiende que la incorporación de las mujeres al análisis de clase pasa por comprender "la importancia relativa de las relaciones directas y mediadas de clase a la hora de determinar sus intereses de clase" (Wright, 1989, p. 42), una tarea que acomete estudiando empíricamente la importancia relativa de ambas relaciones a la hora de dar cuenta de la identidad de clase de las mujeres casadas en Estados Unidos y Suecia.

La publicación de Women in the Class Structure fue el pistoletazo de salida para una consideración más completa de las interconexiones entre las relaciones de clase y las relaciones de género, que se desplegará en esos años con diversos estudios empíricos recogidos luego en una de las partes (la más extensa) de su libro Class Counts (Wright, 2000), que también incluye un planteamiento teórico más sistemático. Wright (2000) afirma que la tarea central del análisis de clase en relación con el género debe ser "explicar, para explananda concretos, las formas de interacción entre la clase y el género como procesos causales" (p. 118) y propone una clasificación conceptual, muy clarificadora, de las posibles interconexiones entre ambas variables. Éstas pueden adquirir cinco formas:

1) El género puede ser una forma de relación de clase, como en el caso en que las mujeres son propiedad de los hombres; 
2) Las relaciones de género y las de clase pueden afectarse reciprocamente, como ocurre cuando la existencia de determinadas posiciones de clase (como el empleo del hogar) es posible sólo gracias a la existencia de un determinado orden de relaciones de género;

3) El género, a través de la socialización diferenciada y los distintos tipos de desigualdad, puede ser un mecanismo de clasificación para determinadas posiciones de clase, dando cuenta, así, de la segregación ocupacional;

4) Las relaciones de género pueden constituir una "conexión mediada" con la estructura de clase, como sucede en el caso de los matrimonios, las familias y el parentesco;

5) y, por último, aparte de sus efectos independientes, el género y la clase pueden tener efectos interactivos (a los que Wright denominaría "clender") a la hora de dar cuenta de determinados fenómenos (Wright, 2000, pp. 118-124).

En esa obra también se incluyen dos estudios empíricos. El primero resume los resultados de su investigación sobre la relación entre la clase social y la división generizada del trabajo doméstico y venía a cubrir el vacío empírico que existía en torno a un tema sobre el que se habían producido muchos debates teóricos en las décadas previas. En particular, el trabajo analizaba comparativamente, a partir de los casos de Suecia y Estados Unidos, cómo variaba la contribución de los maridos al trabajo doméstico en hogares de dos sustentadores en función de la composición de clase del hogar. En contra de sus expectativas teóricas ${ }^{4}$, halló que la posición en la estructura de clase no era un determinante sistemático de las variaciones en la división del trabajo doméstico en los hogares, lo que daba respaldo a la hipótesis feminista de la autonomía de las relaciones de género a la hora de explicar la reproducción de la dominación masculina.

No obstante, el hecho de que encontrara una mayor desigualdad de género en los hogares estadounidenses, especialmente en los puramente de clase media, que en los suecos, parecía apuntar a la importancia del análisis de clase a escala macro-institucional: el grado de igualitarismo existente en los hogares de una sociedad podía estar vinculado, en gran parte, a la movilización política de las clases y, en concreto, a la agenda de género de los actores de la clase trabajadora-argumento en línea con la teoría de los recursos de poder (Huber y Stephens, 2000; Campillo y Sola, 2020)-.

El segundo estudio empírico recoge los resultados de sus investigaciones comparativas en torno a la brecha de género en las posiciones de autoridad en el trabajo y el techo de cristal ${ }^{5}$. Estas perseguían un triple objetivo: describir las variaciones de esas brechas en siete países (Estados Unidos, Canadá, Australia, Reino Unido, Noruega, Suecia y Japón), explorar si adquieren la forma del techo de cristal -esto es, si hay una intensificación de las desventajas que sufren las mujeres según escalan por la jerarquía de autoridad-, e intentar explicar esas variaciones.

En el plano descriptivo, halló que el nivel de desigualdad en el acceso a posiciones de autoridad en el trabajo era menor en los países angloparlantes, bastante mayor en

\footnotetext{
4 En una prueba de su honestidad intelectual, Wright no tiene problema en reconocer que "definitivamente no es lo que esperaba cuando comencé el análisis. De hecho, como parte de mi agenda general de análisis de clase, inicialmente estaba bastante empeñado en demostrar que la clase era una parte importante de la explicación de las variaciones en las prácticas de género. Al principio, cuando encontré estos efectos marginales de la clase, intenté operacionalizar los detalles de la variable de clase de muchas formas alternativas [pero] ninguna de estas manipulaciones de los datos cambió sustancialmente los resultados" (Wright, 2000, pp. 155-156).

5 Para una revisión detallada de sus contribuciones a este campo y una réplica actualizada de sus análisis para el caso de España, véase el artículo de Miguel Caínzos (2021) en este mismo número.
} 
los países escandinavos, y extremadamente grande en Japón. En cuanto a la hipótesis del techo de cristal, los resultados en la mayoría de países no parecían respaldar que las probabilidades de promoción de las mujeres disminuyeran conforme se escalaba por la jerarquía de autoridad.

Por último, tras descartar que la variación en esas brechas de género pudiera atribuirse a diferencias en la composición de la mano de obra de cada país, puso a prueba la importancia explicativa de diversos factores, hallando que la escasez o abundancia relativa de las posiciones de autoridad en la sociedad, y la fortaleza y grado de liberalismo -cristalizado en su énfasis en la igualdad de oportunidades en el empleo- de los movimientos feministas eran los que mejor podían dar cuenta de la variación entre países. De ahí que Estados Unidos, que ofrecía más puestos de autoridad y cuyo movimiento feminista era fuerte y tenía una orientación liberal, tuviera una brecha de género menor, mientras que Suecia, que se caracteriza por una menor proporción de puestos de autoridad y un movimiento feminista de orientación socialdemócrata, centrado más en las provisiones estatales de bienestar que en el sector privado, tuviera una brecha de género mayor.

Esta parte del trabajo de Wright puede contribuir a los debates en boga acerca de la interseccionalidad de la clase, el género y la raza/etnia en los estudios feministas, por más que apenas haya recibido atención -salvo excepciones, como la de Laurel Weldon (2006)- desde este campo. Como han señalado Ann Phoenix y Pamela Pattynama (2006), la interseccionalidad se ha convertido en una "expresión atrapalotodo" (p. 187) rodeada de cierta confusión teórica-metodológica. En su influyente revisión de este campo, Leslie McCall (2005) distingue varios enfoques: por un lado, los estudios de caso que buscan visibilizar la situación de grupos o realidades (como la violencia sobre las mujeres negras) en donde se cruzan varias formas de opresión que los enfoques existentes son incapaces de captar (en ese caso, los estudios de género centrados en la situación de las mujeres blancas y los estudios de raza/etnia centrados en los hombres negros); por el otro, los estudios cuantitativos que analizan las múltiples y cambiantes formas de desigualdad en función de diferentes variables (clase, género, etnia/raza u otros).

Pues bien, en línea con las ideas de McCall, el trabajo de Wright puede ser un buen punto de apoyo tanto para la clarificación analítico-conceptual de muchos debates sobre la interseccionalidad como para diseñar estrategias metodológicas para estudiarla empíricamente. En términos estadísticos, la interseccionalidad puede identificarse con los efectos interactivos de dos (o más) variables, que pueden añadirse a sus efectos aditivos ${ }^{6}$, pero el menú de cinco posibles interconexiones entre género y clase también puede servir como punto de partida para estudios de caso o análisis comparativos. En ese sentido, la claridad teórico-conceptual para formular del modo más preciso posible los diferentes procesos causales que intervienen en las conexiones entre las relaciones de clase y las de género (a las que se puede añadir las de raza/etnia u otras más) puede ser un buen antídoto contra la vaguedad que con frecuencia sobrevuela estos debates.

En resumen, el trabajo de Wright sobre las interconexiones entre clase y género supuso un avance en la comprensión de algunos fenómenos (Caínzos, 2021), pero continúa siendo inspirador por, al menos, tres razones: la primera es su capacidad para deslindar teóricamente diferentes tipos de procesos causales que pueden estar en juego (como los recogidos en su menú); la segunda es su rechazo a adoptar apriorismos teóricos fuertes para fenómenos que deben contrastarse empíricamente (como el peso de los efectos de las relaciones directas y mediadas) o su disposición a aceptar resultados que contradicen sus expectativas teóricas (como en el caso de la ausencia de efectos en la división del

\footnotetext{
6 McCall (2005) añade a los efectos interactivos de dos variables (por ejemplo, clase y género) otra variable que resultaría de fusionar ambas, pero en realidad -al menos, en función de cómo se operacionalice- ambas opciones estarían midiendo lo mismo (en este caso, lo que Wright llama "clender").
} 
trabajo doméstico en función de la clase); y la tercera es su saludable ecumenismo o "realismo pragmático" -visible en sus últimos trabajos (Wright, 2005, 2015), en los que desafortunadamente apenas dedicó atención al género-, que invita a no sobredimensionar las diferencias teóricas y buscar puntos de encuentro entre posturas en disputa.

\section{UTOPÍAS FEMINISTAS: IGUALITARISMO FUERTE Y SOCIEDAD SIN GÉNERO}

Como señalaba, a principios de los años noventa Wright comenzó a interesarse por la reflexión sobre las alternativas emancipadoras al capitalismo, y en los años siguientes esas utopías reales desplazaron al análisis de clase del centro de su trabajo. Ese cambio de programas de investigación se ha interpretado a veces como un salto entre dos proyectos independientes y desconectados (Burawoy, 2020), pero es posible rastrear las continuidades entre ambos y entender el segundo como una respuesta a los problemas e interrogantes que había suscitado el primero (Krippner, 2019; Seidman, 2020).

Para Wright, el interés por el estudio de la estructura de clases de las sociedades capitalistas avanzadas había radicado en su convicción de que representaba una condición necesaria para comprender los procesos de formación de actores políticos de clase y proponer, de este modo, un proyecto socialista viable. No obstante, como señala Greta Krippner (2019), “conforme el proyecto empírico de Erik se fue haciendo más ambicioso y su mapa de las posiciones de clase, más elaborado, comenzó a albergar dudas cada vez mayores de que su trabajo pudiera cosechar los frutos políticos que había anticipado inicialmente"7 (p. 4). Igualmente, el reconocimiento de la importancia de las posiciones mediadas de clase en la experiencia vital de la gente llevó a Wright a replantearse tanto los objetivos emancipadores del socialismo como las vías posibles para alcanzarlos (Seidman, 2020, p. 14). A principios de los noventa, este reconocimiento coincidía con la desintegración del bloque soviético y el descrédito de los enfoques más deterministas de la tradición marxista.

El artículo "Explanation and Emancipation in Marxism and Feminism" refleja su creciente preocupación por "desarrollar una teoría más positiva y más sistemática de la sociedad sin clases" (Wright, 1993, p. 54) que fuera capaz de relanzar el proyecto emancipador socialista en ese contexto adverso. Pero es también una prueba de la influencia que tuvo el feminismo en esta empresa: el objetivo era contrastar ambas tradiciones de teoría social emancipadora, con el fin de "precisar mejor nuestra comprensión de los dilemas a los que se enfrenta el marxismo hoy" (Wright, 1993, p. 39).

Más en concreto, el feminismo le sirve como espejo para reflexionar sobre los motivos por los que la utopía socialista no tiene ya el apoyo o la validez que sí tiene la utopía feminista: Wright subraya que, frente al escepticismo creciente de marxistas y personas de izquierdas en general en relación a la viabilidad de una sociedad sin clases, las feministas de diversas corrientes nunca han dudado de la viabilidad de una sociedad libre de la opresión de género. Según Wright, esto es así porque "la clase y el género difieren en términos de su relación entre las micro-experiencias vividas en las relaciones existentes y los cambios macro-institucionales necesarios para la emancipación" (Wright, 1993, p. 46). Dicho de otro modo, incluso las relaciones desiguales de género actuales "contienen elementos que prefiguran simetría e igualdad": en el movimiento feminista o en la vida familiar, las mujeres tienen experiencias de "reciprocidad y compañerismo que prefiguran la posibilidad de relaciones igualitarias, si bien de forma limitada" (Wright, 1993, p. 46).

7 Krippner (2019, p. 4) subraya, a este respecto, las palabras de Wright (1987) en una nota al pie en The Debate on Classes: "Aunque sigo pensando que resolver los problemas conceptuales en el análisis de la estructura de clases es importante, ya no creo que sea la clave para entender el problema más general de las variaciones en la formación de clase y de las posibilidades de creación de coaliciones radicales". 
En cambio, sostiene Wright, es mucho más difícil extrapolar de lo micro a lo macro en la cuestión de clase porque las experiencias que prefiguran futuros emancipadores en el caso de los trabajadores se dan sólo entre trabajadores (y no con los empresarios); y porque la producción socialista requiere una coordinación activa a escala macro (mecanismos institucionales de planificación, flujos de información, distribución del capital, fijación de precios) cuyas dificultades siembran dudas sobre su viabilidad ${ }^{8}$.

Sea como fuere, el hecho es que el proyecto emancipador marxista ha dependido tradicionalmente de unas teorías deterministas de la historia, mientras que el proyecto feminista ha estado mucho más centrado en la agencia. Una vez agotada la credibilidad de esas teorías, era preciso aprender del feminismo y reflexionar sobre las posibles vías de desarrollo de un proyecto socialista. De este reconocimiento nace el proyecto al que se dedicó en sus últimos veinticinco años: las utopías reales (Sola, 2021).

En una época de desencanto y escepticismo ante los proyectos emancipadores de cambio social, el objetivo de este proyecto era "contribuir a la reconstrucción de la posibilidad del cambio social emancipador investigando la factibilidad de instituciones de tipo radicalmente distinto y de relaciones sociales que podrian hacer avanzar los objetivos democráticos igualitarios históricamente asociados con la idea del socialismo" (Wright, 2014 , p. 17). No se trataba de formular grandes modelos, sino de plantear "propuestas eficaces capaces de mejorar de forma pragmática nuestras instituciones" y de aumentar "el bienestar y la felicidad humanos" (Wright, 2014, pp. 8-22). Estas propuestas, basadas en el diagnóstico crítico de las instituciones y estructuras sociales existentes, debían ser normativamente deseables, técnicamente viables y políticamente factibles, lo que incluía prestar atención a sus posibles consecuencias no intencionadas.

A lo largo de los años, el proyecto se desarrolló de la siguiente manera: Wright reunía periódicamente en Madison a una serie de investigadores que reflexionaban y debatían en torno a alguna propuesta de "utopía real" elaborada por uno o varios de ellos. Todos los textos, tanto el de la propuesta central como las distintas réplicas, eran recogidos y editados posteriormente por Wright en la editorial Verso. En total, se han publicado siete libros que han abordado propuestas relacionadas con la democracia asociativa (Cohen y Rogers, 1995), el socialismo de mercado (Roemer, 1996), los diseños institucionales igualitarios (Bowles y Gintis, 1998), la democracia participativa (Fung y Wright, 2003), la renta básica y el capital básico (Ackerman et al., 2006), la igualdad de género (Gornick y Meyers, 2009), y el uso político del sorteo (Gastil y Wright, 2019).

Pese a que el pensamiento feminista marcó el viraje de Wright hacia la reflexión acerca de las alternativas emancipadoras al capitalismo y la importancia estratégica que le concedió a la comunidad (el "poder social" o "asociativo") y los valores compartidos (Wright, 2014, 2019), lo cierto es que no reflexionó en profundidad ni de modo sistemático sobre cómo sería una utopía real relacionada con la igualdad de género o sobre el papel que desempeñaría la igualdad de género en las estrategias de transformación hacia una sociedad postcapitalista. De hecho, en Construyendo utopías reales (Wright, 2014), en el que contextualiza, justifica y sintetiza el trabajo realizado en los años previos en el marco del proyecto de las utopías reales, apenas cita el libro colectivo sobre igualdad de género en torno a la propuesta de Gornick y Meyers (2009) ni recoge ese debate, mientras que sí se abordan extensamente las discusiones sobre el resto de propuestas utópico-realistas.

8 En el contraste de estas dos tradiciones emancipadoras, las posibilidades y dilemas asociados a la emancipación feminista resultan un tanto simplificadas. Por un lado, Wright ofrece una imagen sesgada positivamente de las posibilidades que tienen las mujeres para vivir experiencias de simetría e igualdad, especialmente en la familia; por otro lado, considera que el problema del patriarcado se juega principalmente en los "escenarios micro de las prácticas de género", que "no hay contradicciones distintivas de género en la coordinación macro como tal", pues en las relaciones de género la cuestión macro fundamental está relacionada con la intervención del Estado en el nivel micro (si bien admite que esa extrapolación podría ser ilegitima si resultara que la reproducción del capitalismo o de la sociedad fuera incompatible con la eliminación de la opresión de género). 
Con todo, sí que dedicó algunos textos a reflexionar sobre la desigualdad de género, los objetivos de una política feminista y los mecanismos institucionales para lograrlos. Los planteamientos que aparecen en esos trabajos no forman un todo coherente y sistemático y, en algunos casos, van cambiando con el tiempo. A continuación, se revisarán fundamentalmente dos aspectos de esa reflexión: por un lado, su visión inicial de la opresión de genero y sus propuestas institucionales para lograr la igualdad de género; por el otro, su defensa posterior de una sociedad sin género (genderlessness society).

En cuanto a lo primero, inicialmente Wright diferencia el "género", entendido como la construcción social de la diferencia sexual, de la "opresión de género", que se definiría como "una situación en la que las categorías relevantes de actores sociales (...) difieren en poder social y en bienestar material" (Wright, 1993, p. 41) o como una situación desigual "en términos de poder, recompensas y cargas" (Brighouse y Wright, 2009, p. 82). Por tanto, una utopía real igualitaria en términos de género implicaría "eliminar los diferenciales de poder y bienestar entre hombres y mujeres" (Wright, 1993, p. 41) o, dicho de otro modo, supondría que "las relaciones de género (...) no generan oportunidades y opciones desiguales para hombres y mujeres" (Wright y Rogers, 2011, p. 298).

Aunque no hay una reflexión del todo explícita sobre ello, Wright da a entender que lo contrario de la opresión de género es la igualdad de género, que algunas veces parece asimilar a un proyecto de "justicia de género" (Fraser, 2013) y otras veces se acerca más bien hacia un proyecto de "simetría de género" (Orloff, 2009). Como veremos, esta segunda visión del proyecto igualitario de género será la que acabará predominando en sus reflexiones.

Ahora bien, ¿en qué se basan los diferenciales de poder y bienestar entre hombres y mujeres? O dicho de otro modo, ¿cuál es, para Wright, la fuente del patriarcado? Pese a que reconoce que la desigualdad de género se expresa en distintos campos (Wright y Rogers, 2011) y que las feministas difieren considerablemente en el análisis sobre sus causas, que algunas sitúan en las prácticas culturales y sexuales que moldean subjetividades y expectativas diferenciadas, y otras en las instituciones económicas y políticas de poder y privilegio (Wright, 1993), lo cierto es que -en línea con la tradición del feminismo socialistaWright sitúa el núcleo del patriarcado en la división sexual del trabajo en la familia.

De ahí que afirme que un fuerte igualitarismo de género significa "una estructura de relaciones sociales en la que la división del trabajo doméstico y de cuidados en la familia y las distribuciones ocupacionales en el espacio público no estén afectados por el género" (Brighouse y Wright, 2009, p. 82) y que "para acercarnos por completo a la idea de igualdad de género, quizá el problema clave que necesitamos resolver es la transformación de la división generizada del trabajo en la familia" (Wright y Rogers, 2011, p. 326). No obstante, como veremos, las reflexiones y propuestas de Wright en torno a la división sexual del trabajo doméstico y de cuidados en las familias se centran fundamentalmente en la cuestión de la crianza, olvidando las tareas domésticas y otro tipo de cuidados menos gratificantes (Folbre, 2009).

La primacía de la división sexual del trabajo de la crianza en su análisis de la desigualdad de género se traslada al tipo de mecanismos institucionales y políticas públicas que propone para avanzar el proyecto de una utopía real igualitaria en términos de género: igualdad salarial para trabajos de equivalente o de igual valor, universalización de unos servicios de atención y educación infantil de alta calidad, y permisos parentales remunerados igualitarios (Wright y Rogers, 2011) ${ }^{9}$. Sin duda, Wright entendía que esta última política era

\footnotetext{
9 No está claro cuánto hay de utópico en estas propuestas que toman como modelo políticas que ya se han desarrollado, hasta cierto punto, en algunos países escandinavos, en los que, sin embargo, no se ha alcanzado la igualdad de género. Como el propio Wright había estudiado, Suecia tiene políticas de igualdad en el campo del bienestar y la crianza bastante desarrolladas y, aun así, presenta una mayor brecha de género en las posiciones de autoridad en el trabajo que EEUU. Lo que apunta, como subraya Orloff (2009) en su contribución al monográfico sobre igualdad de género del proyecto Utopías Reales, a que "quizás otras fuerzas -no sólo la división generizada del trabajo- están implicadas en el mantenimiento de la jerarquía de género" (p. 133).
} 
la que mayor potencial tenía para impulsar un proyecto de "igualitarismo fuerte de género" (Brighouse y Wright, 2009).

La defensa de los permisos parentales iguales e intransferibles de 6 meses era, junto a la reducción de la jornada semanal a entre 35 y 39 horas y la universalización de los servicios de atención y educación infantil, el núcleo de la propuesta de Gornick y Meyers (2009) que ocupó el encuentro y el libro del proyecto de Utopías Reales dedicado a la igualdad de género. En su contribución a ese debate, Brighouse y Wright defienden un esquema de permisos individuales que promuevan la igualdad -entendida como una distribución simétrica en términos de género de la crianza en el interior de la familia (heterosexual)- de forma más "radical": tras un permiso de un mes para su recuperación física del parto, las madres podrían disfrutar de un permiso remunerado adicional cuya duración, hasta un máximo de seis meses, sería igual a la del permiso al que se acogiera el padre. Es decir, si el padre decidía cogerse sólo dos meses de permiso, la madre no podría disfrutar más que dos meses, de modo que había un incentivo familiar para que el padre aprovechara al máximo su derecho.

Esta propuesta sería deseable, a juicio de Brighouse y Wright, porque "en familias con hijos, las perspectivas para el florecimiento tanto de hombres como de mujeres aumentarian, en general, si las actividades asociadas al cuidado y la crianza de las criaturas se repartieran más igualitariamente" y porque "las cargas y responsabilidades extra que soportan las mujeres en la familia constriñen su capacidad para competir en el mercado laboral" y "refuerzan estereotipos sobre los compromisos laborales y las prioridades de las mujeres" (Brighouse y Wright, 2009, p. 81). El hecho de que el esquema propuesto fuera en contra "de la idea central del feminismo de potenciar la autonomía de las mujeres y aumentar su rango de opciones independientemente de los varones" (Brighouse y Wright, 2009, p. 90) era considerado un mal menor frente al objetivo central del igualitarismo fuerte de género de alentar activamente a los hombres a comprometerse más en la crianza.

La propuesta no sólo desprecia la voz y la autonomía de las mujeres, sino que pasa por alto que cada vez más criaturas crecen en hogares monoparentales ${ }^{10}$-encabezados mayoritariamente por mujeres- $u$ homoparentales. Asimismo, parece dar por hecho que un esquema así sería exitoso porque los varones se tomarían el máximo de meses posible. No deja de resultar sorprendente que Wright apostase con entusiasmo por una propuesta que no cumplía ni con algunas exigencias normativas de justicia ni con ciertas precauciones prácticas que él mismo reconocía que debían guiar una ciencia social emancipadora. El planteamiento de un esquema de permisos de este tipo obvia tanto el principio democrático básico de que todo el mundo tenga igual capacidad para participar en decisiones colectivas que afectan a su vida, como los requisitos de factibilidad política y anticipación de las consecuencias no intencionadas que debían cumplir las utopías reales propuestas.

En cuanto al segundo aspecto de su reflexión en torno a una utopía real relacionada con el género, Wright (2011) desarrolla la idea de una "sociedad sin género", en oposición a la "igualdad de género", y la defiende como el horizonte al que dirigirnos. Si bien esta postura entronca con la propuesta comentada anteriormente, lo cierto es que supone un paso más allá en su comprensión del género y la opresión de género. Wright (2011) sostiene aquí que "las relaciones de género son inherentemente coercitivas en el sentido de que imponen limitaciones socialmente impuestas a las elecciones y prácticas de hombres y mujeres" (p. 403).

Esta identificación entre género y opresión de género le lleva a afirmar que el objetivo emancipador no debería ser la igualdad de género, entendida como una situación en la que

10 Según el Pew Research Center, en 2019 un cuarto de las personas menores de 18 años en EE. UU. vivía en hogares monoparentales. 
"las desigualdades de ingresos, poder, y estatus no estuvieran ya asociadas con el género" (Wright, 2011, p. 412), sino la completa disolución del género (genderlessness), ya que la mera existencia de relaciones y expectativas de género "aún socavaría el igual acceso al florecimiento humano para aquellas personas, hombres o mujeres, con las disposiciones 'equivocadas'" (Wright, 2011, pp. 412-413). Al igual que el ideal normativo socialista es una sociedad sin clases, el ideal igualitarista en términos de género debería ser, pues, una sociedad sin género. Esta utopía se basaría presumiblemente en lo que Orloff (2009) llama el principio de la simetría "en la asignación y desempeño del trabajo de cuidados y del trabajo remunerado ( $y$, presumiblemente, otros tipos de participación en las actividades colectivas, como la política)" (p. 129).

La propuesta de Wright entronca, por un lado, con una cierta línea de pensamiento feminista liberal que parte del modelo ilustrado del "yo desvinculado" (Sandel, 1984) y defiende que una sociedad justa sería aquella en la que ser hombre o mujer fuera tan poco significativo como "el color de los ojos o la longitud de los dedos de los pies" (Okin, 1991 p. 171) ${ }^{11}$. Por otro lado, aunque no se extiende en los cambios institucionales y las estrategias políticas que podrían conducir a esa sociedad desgenerizada, de su reflexión se desprende una visión que minimiza los conflictos de intereses entre hombres y mujeres y tiende a atribuir a ambos unos intereses "reales" (y homogéneos) que no tienen por qué coincidir con los que realmente tienen (ver las críticas a esta visión por parte de Orloff, 2009; Folbre, 2009; Shalev, 2009). De este modo, la estrategia de transformación hacia la sociedad sin género se presenta como poco problemática.

Sin duda, la abolición del género es una idea atractiva en términos abstractos, pero plantea serios problemas prácticos. Por usar el instrumental analítico del propio Wright, podríamos dudar de su viabilidad y su deseabilidad. En primer lugar, como han señalado algunas feministas, la idea de una sociedad sin género parece encajar mal con nuestra experiencia psicológica estrechamente vinculada a la diferencia sexual (Nussbaum, 1992). Igualmente, resulta problemático pensar que los efectos normativos de esa diferencia desaparecerian por completo, como defiende Wright (2011) al sostener que, pese a que podría haber alguna "correlación entre el sexo de una persona y su rol social"12, eso "no generaría expectativas normativamente respaldadas sobre quién debería hacer qué" ( $p$. 405). En este sentido, la idea de una sociedad sin género resulta "problemática si de lo que estamos hablando es de utopías reales" -por usar la expresión, sacada de otro contexto, de Orloff (2009, p. 152)-.

En segundo lugar, aún cuando la idea resultara viable, podría cuestionarse su deseabilidad a la luz de las posibles consecuencias no intencionadas. Wright señala que las diferencias en las disposiciones y preferencias personales serían contingentes en términos de género, pero no aborda con detenimiento la cuestión de hasta qué punto se acercarían a uno de los modelos previamente existentes. Es más, en relación a la crianza, señala que en una sociedad sin género la distribución de las disposiciones de hombres y mujeres respecto al cuidado tendería a confluir en una posición intermedia respecto a la distribución bimodal actual, y que la distribución de los comportamientos de los hombres se aproximaría a la distribución actual de las mujeres.

\footnotetext{
11 Como sostiene Orloff (2009), es esta una concepción del género muy unidimensional, que lo concibe únicamente como coacción, y que pasa por alto "las profundas inversiones que las personas tienen en el género, y los modos en que el conocimiento, la subjetividad y la agencia se ven limitados y posibilitados por las categorías de género existentes" (pp. 137-138). La identificación de género con opresión de género pasa por alto, además, que el reconocimiento de las diferencias sexuales y reproductivas debe ser tenido en cuenta en una sociedad justa y no tendría por qué implicar jerarquía de género (Haslanger, 2012).

12 “Por ejemplo, por razones biológicas, es inherentemente más fácil que una mujer soltera se convierta en madre que un hombre soltero se convierta en padre, y, como resultado, habrá ciertamente más mujeres que hombres activamente criando, incluso en ausencia de normas coercitivas de género" (Wright, 2011, p. 405).
} 
Pero hay razones para pensar que el resultado sería justo el contrario ${ }^{13}$. El proceso de incorporación de la mujer al mercado laboral durante el último medio siglo ha propiciado, en un contexto de remercantilización generalizada, un cambio en los estilos de vida de las mujeres tendente a priorizar las exigencias del empleo asalariado sobre las necesidades de cuidado, sin que los estilos de vida de los hombres hayan cambiado en dirección opuesta. Como reflexiona la socióloga Arlie Hochschild (2009): “las mujeres como yo han deseado ser iguales a los hombres en la vida pública y en la vida privada. Pero este deseo suscita una pregunta: ¿iguales a qué? ¿lguales en qué cultura del cuidado? (...) Muchas mujeres hemos emigrado de la cultura de nuestra madre para establecernos en la cultura de nuestro padre (...) ¿Qué hemos dejado atrás? ¿Nos sentimos bien con lo que tenemos?" (p. 19). En realidad, -como sugiere la evidencia histórico-antropológica (Hernando, 2012)- tampoco resulta obvio que toda diferencia de género deba implicar una jerarquía.

Dicho esto, la reflexión en torno a los problemas de viabilidad y deseabilidad de una sociedad sin género puede servirnos como cautela a la hora de emprender un camino, más realista, hacia una sociedad en la que el género desempeñe un papel menos importante en las disposiciones y preferencias de las personas y estas lo experimenten con mayor fluidez que en la actualidad.

En resumen, el feminismo ejerció su influencia en el viraje de Wright hacia la reflexión sobre las alternativas emancipadoras al capitalismo, y su proyecto de las utopías reales prestó cierta atención, si bien irregular, a la igualdad de género. Wright aportó ideas originales (y ciertamente audaces), por más que llevaran aparejadas serios problemas como los que he señalado. Pero no profundizó en una utopía real "feminista", quizás por una inhibición personal para internarse en un territorio en el corría el peligro de hablar por las mujeres desde su privilegio como hombre. Con todo, las herramientas teóricoconceptuales que Wright elaboró para este programa de investigación pueden servir para valorar medidas utópico-realistas en relación al género ${ }^{14}$, y sus reflexiones sobre la opresión de género y su disolución son contribuciones valiosas a debates y dilemas que siguen abiertos en el feminismo actual.

\section{A MODO DE CONCLUSIÓN}

Este artículo ha ofrecido una revisión de la obra de Wright desde una perspectiva feminista, señalando su creciente atención al género y la influencia que ejerció el feminismo en su trayectoria. Por un lado, los debates feministas contribuyeron al desarrollo de una de sus principales aportaciones al análisis de clase -la formulación teórica de las posiciones mediadas de clase y la exploración empírica de su importancia relativa-y, más adelante, a una reflexión más sistemática de las interacciones entre clase y género. En este terreno mostró su característica capacidad para la clarificación teórico-conceptual de los procesos causales que subyacen a los fenómenos sociales, de la que podrían beneficiarse los estudios y debates actuales sobre la interseccionalidad.

Por otro lado, el reconocimiento de la importancia de las relaciones familiares y comunitarias en la formación de la identidad y los valores de la gente, así como la creciente complejidad de su mapa de posiciones de clase, le llevaron a abandonar progresivamente ese programa de investigación para dedicarse a la reflexión en torno a los objetivos

13 Con su prudencia habitual, Wright (2011) admite que se trata de una hipótesis sin respaldo empírico y que existe la posibilidad de
que en un mundo desgenerizado "los modelos masculinos actuales se generalizaran a todas las personas" (p. 408), pero no se toma que en un mundo desgenerizado "los modelos
suficientemente en serio esta posibilidad.

14 Véase, como ejemplo, el especial sobre feminismo y renta básica en el número 3(3) de la revista Basic Income Studies. 
emancipadores del socialismo y a las vías para alcanzarlos, con su proyecto sobre las utopías reales.

Ese proyecto incluyó una propuesta y debate en torno a la igualdad de género (Gornick y Meyers, 2009), pero la atención que recibió este tema en la obra posterior de Wright (2014, 2019) fue relativamente menor. Con todo, en sus últimos años dedicó algunos trabajos puntuales a reflexionar sobre la desigualdad de género, los objetivos de una política feminista y los mecanismos institucionales para lograrlos, cuyas ideas principales hemos revisado. Más en concreto, se ha puesto de relieve el cambio en su visión de la opresión de género y, con él, la transformación de su ideal normativo en este terreno: de la igualdad de género (en el acceso a los recursos de bienestar y poder) a la abolición completa del género. Como se ha argumentado, tanto en esa propuesta, de naturaleza básicamente teórica, como en su posición respecto a los permisos parentales, Wright parece dejarse llevar por un excesivo optimismo de la inteligencia y relaja sus precauciones acerca de las consecuencias no intencionadas del cambio social. No obstante, su postura conecta en ambos casos con una cierta tradición de pensamiento feminista y saca a la luz dilemas y problemas de los que se sigue discutiendo actualmente en el feminismo.

Como ha subrayado Gay Seidman (2020), la creciente atención de Erik Olin Wright a las relaciones de género y sus ideas (y sesgos, podría añadirse) con respecto a una utopía igualitaria en términos de género no puede entenderse sin hacer mención a su vida privada, sus experiencias familiares y sus amistades. Utilizando la terminología de Gilligan (1982), podríamos decir que Wright aunaba en su persona la ética universalista de los derechos y la ética del cuidado y la responsabilidad. Como él mismo reflexionaba en una de las últimas entradas de Caringbridge, el blog en el que iba recogiendo reflexiones y recuerdos varios junto a las noticias sobre su enfermedad: "Veo el amor como un hilo profundo en mi vida, que unifica cómo enseño, cómo soy como padre, mi trabajo académico y mis compromisos con el marxismo y la ciencia social emancipadora" (Wright, 2020).

\section{AGRADECIMIENTOS}

Una versión previa de este artículo se presentó, en forma de ponencia, en las Jornadas Homenaje a Erik Olin Wright celebradas en enero de 2020 en la Universidad Complutense de Madrid y la UNED. Agradezco los comentarios de los participantes en tales jornadas, especialmente los de Miguel Caínzos, Julio Carabaña, José Antonio Noguera, José Saturnino Martínez, Olga Salido, Leire Salazar y Andrés de Francisco. Debo, además, reconocer mi deuda especialmente con dos personas: Gay Seidman, por hacerme llegar generosamente el borrador de su ponencia "Class, gender and utopian community", que fue la semilla a partir de la cual creció este trabajo, y por sus comentarios a mis primeras ideas; y Jorge Sola, sin cuyos apuntes y labor de edición este artículo sería mucho peor.

\section{REFERENCIAS}

Acker, J. (1973). Women and Social Stratification: A Case of Intellectual Sexism. American Journal of Sociology, 78(4), 936-945.

Ackerman, B. A., Alstott, A. y Van Parijs, P. (2006). Redesigning distribution: Basic income and stakeholder grants as alternative cornerstones for a more egalitarian capitalism. Londres: Verso. 
Albright, K. (2008). In Families or as Individuals? Theoretical and Methodological Problems in the Incorporation of Women in Class Analysis. Sociology Compass, 2(5), 1672-1689. https://doi.org/10.1111/j.1751-9020.2008.00156.x

Baxter, J. y Wright, E. O. (2000). The Glass Ceiling Hypothesis: A Comparative Study of the United States, Sweden, and Australia. Gender \& Society, 14(2), 275-294. https://doi. org/10.1177/089124300014002004

Bowles, S. y Gintis, H. (1998). Recasting egalitarianism: New rules for communities, states, and markets. Londres: Verso.

Brighouse, H. y Wright, E. O. (2009). Strong Gender Egalitarianism. En J. C. Gornick y M. Meyers (eds.), Gender equality: Transforming family divisions of labor (pp. 79-92). Londres: Verso.

Burawoy, M. (2020). A Tale of Two Marxisms. New Left Review, 121, 66-98.

Caínzos, M. (2021). Desigualdad persistente, pero no un techo de cristal. Sobre género y autoridad en el trabajo. Revista Española de Sociología, 30(2), a43. https://doi. org/10.22325/fes/res.2021.43

Campillo, I. y Sola, J. (2020). La teoría de los recursos de poder: una revisión crítica. Revista Española de Investigaciones Sociológicas, 170, 19-34. http://dx.doi.org/10.5477/cis/ reis.170.19

Cohen, J. y Rogers, J. (1995). Associations and democracy. Londres: Verso.

Delphy, C. (1984). Close to Home: A Materialist Analysis of Women's Oppression. Amherst: University of Massachusetts Press.

Folbre, N. (2009). Reforming Care. En J. C. Gornick y M. Meyers (eds.), Gender equality: Transforming family divisions of labor (pp. 111-128). Londres: Verso.

Fraser, N. (2013). Fortunes of Feminism. From State-Managed Capitalism to Neoliberal Crisis. Londres: Verso.

Fung, A. y Wright, E. O. (2003). Deepening Democracy: Institutional Innovations in Empowered Participatory Governance. Londres: Verso.

Gastil, J. y Wright, E. O. (2019). Legislature by Lot. Transformative Designs for Deliberative Governance. Londres: Verso.

Gilligan, C. (1982). In a different voice. Psychological Theory and Women's Development. Cambridge: Harvard University Press.

Goldthorpe, J. H. (1983). Women and Class Analysis: In Defense of the Conventional View. Sociology, 17(4), 465-488. https://doi.org/10.1177/0038038583017004001

Goldthorpe, J. H. (1984). Women and Class Analysis: A Reply to the Replies. Sociology, 18(4), 491-499. https://doi.org/10.1177/0038038584018004002

Gornick, J. C. y Meyers, M. (2009). Gender equality: transforming family divisions of labor. Londres: Verso.

Hartmann, H. I. (1979). The Unhappy Marriage of Marxism and Feminism: Towards a more Progressive Union. Capital \& Class, 3(2), 1-33.

Haslanger, S. (2012). Resisting Reality: Social Construction and Social Critique. Oxford: Oxford University Press.

Heath, A. y Britten, N. (1984). Women's Jobs do Make a Difference: A Reply to Goldthorpe. Sociology, 18(4), 475-490. 
Hernando, A. (2012). La fantasía de la individualidad: sobre la construcción sociohistórica del sujeto moderno. Buenos Aires: Katz.

Hochschild, A. (2009). La mercantilización de la vida íntima. Apuntes de la casa y el trabajo. Buenos Aires: Katz.

Huber, E. y Stephens, J. (2000). Partisan Governance, Women's Employment, and the Social Democratic Service State. American Sociological Review, 65(3), 323-342. https://doi. org/10.2307/2657460

Krippner, G. (2019). Comment on Michael Burawoy's “Erik Olin Wright: A Tale of Two Marxisms". Trabajo presentado en Erik Olin Wright Festschrift, Madison.

McCall, L. (2005). The Complexity of Intersectionality. Signs, 30(3), 1771-1800.

Nussbaum, M. (8 de octubre de 1992). Justice for Women! The New York Review of Books. Recuperado de https://www.nybooks.com/articles/1992/10/08/justice-for-women/

Okin, S. M. (1991). Justice, Gender, and the Family. Nueva York: Basic Books.

Orloff, A. (2009). Should feminists aim for gender symmetry? Why a dual-earner/dualcaregiver society is not every feminist's utopia. En J. C. Gornick \& M. Meyers (eds.), Gender equality: Transforming family divisions of labor (pp. 129-160). Londres: Verso.

Phoenix, A. y Pattynama, P. (2006). Intersectionality. European Journal of Women's Studies, 13(3), 187-192. https://doi.org/10.1177/1350506806065751

Roemer, J. E. (1989). Teoría general de la explotación y de las clases. Madrid: Siglo XXI.

Roemer, J. E. (1996). Equal shares: Making market socialism work. Londres: Verso.

Sandel, M. (1984). The Procedural Republic and the Unencumbered Self. Political Theory 12(1), 81-96. http://www.jstor.org/stable/191382

Seidman, G. (2020). Class, gender and utopian community: In memory of Erik Olin Wright. Trabajo presentado en Congreso Transforming Capitalism Through Real Utopias, Coimbra (Portugal).

Shalev, M. (2009). Class Divisions among Women. En J. C. Gornick \& M. Meyers (eds.), Gender equality: Transforming family divisions of labor (pp. 255-282). Londres: Verso.

Sola, J. (2021). Las utopías reales de Erik Olin Wright: ¿una reconstrucción científica del socialismo utópico? Revista Española de Sociología, 30(2), a42. https://doi.org/10.22325/ fes/res.2021.42

Stanworth, M. (1984). Women and Class Analysis: A Reply to John Goldthorpe. Sociology, 18(2), 159-170. https://doi.org/10.1177/0038038584018002001

Weldon, S. (2006). The Structure of Intersectionality: A Comparative Politics of Gender. Politics \& Gender, 2(2), 235-248. https://doi.org/10.1017/S1743923X06231040

Wright, E. O. (1979). Class Structure and Income Determination. Nueva York: Academic Press

Wright, E. O. (1983). Clase, crisis y Estado. Madrid: Siglo XXI.

Wright, E. O. (1987). Rethinking, Once Again, the Concept of Class Structure. En E. O. Wright, U. Becker, J. Brenner, M. Burawoy, V. Burris, G. Carchedi..., P. Van Parijs, The Debate on Classes (pp. 260-347). Londres: Verso.

Wright, E. O. (1989). Women in the Class Structure. Politics \& Society, 17(1), 35-66. https://doi. org/10.1177/003232928901700102 
Wright, E. O. (1993). Explanation and Emancipation in Marxism and Feminism. Sociological Theory, 11(1), 39-54.

Wright, E. O. (1994a). Clases. Madrid: Siglo XXI.

Wright, E. O. (1994b). Interrogating Inequality: Essays on Class Analysis, Socialism and Marxism. Londres: Verso.

Wright, E. O. (2000). Class counts: Comparative studies in class analysis. Cambridge: Cambridge University Press.

Wright, E. O. (2005). Approaches to Class Analysis. Cambridge: Cambridge University Press.

Wright, E. O. (2011). In defense of genderlessness. En A. Gosseries \& P. Vanderborght (eds.), Arguing about justice (pp. 403-413). Lovaina: Presses universitaires de Louvain.

Wright, E. O. (2014). Construyendo utopías reales. Madrid: Akal.

Wright, E. O. (2015). Understanding class. Londres: Verso.

Wright, E. O. (2019). How to be an anticapitalist in the twenty-first century. Londres: Verso.

Wright, E. O. (2020). Christmas-January 12th [en línea]. Recuperado de https://www. caringbridge.org/visit/erikolinwright/journal

Wright, E. O., Baxter, J. y Bikelund, G. E. (1995). The gender gap in workplace authority: A cross-national study. American Sociological Review, 60 (3), 407-35. https://www.jstor. org/stable/2096422?seq=1

Wright, E. O. y Baxter, J. (2000). The Glass Ceiling Hypothesis: A Reply to Critics. Gender \& Society, 14(6), 814-821. https://doi.org/10.1177/089124300014006008

Wright, E. O. y Rogers, J. (2011). American society: How it really works. New York: W. W. Norton \& Company.

Wright, E. O., Shire, K., Hwang, S.-L., Dolan, M. y Baxter, J. (1992). The non-effects of class on the gender division of labor in the home: A Comparative Study of Sweden and the United States. Gender \& Society, 6(2), 252-282.

\section{NOTA BIOGRÁFICA}

Inés Campillo es doctora en Sociología y profesora ayudante doctora en el Departamento de Sociología Aplicada de la Universidad Complutense de Madrid. 